fibrin results in an inflammatory process that effectively damages the capillaries of a capsule, increasing the permeability of the vascular wall and producing bleeding from dilated microvessels beneath the fibrous capsule. In some cases, erosion of microvessels may cause massive hemoptysis. Despite the calcification of the capsule, it seems that a small degree of elasticity exists, which is the reason hematomas usually expand over a long period of time.

These lesions are difficult to differentiate from soft-tissue sarcomas or other malignancies because many of them also reveal hemorrhagic and cystic changes on radiology. ${ }^{2-4}$ The best diagnostic tools are computed tomography and magnetic resonance imaging, including guided fine-needle biopsy.

Hanagiri and colleagues ${ }^{4}$ reported that palliative procedures, such as removal of the inner substance without whole capsule excision, may produce uncontrollable bleeding or recurrence of the hematoma within several years. In cases such as the one reported, the authors believe that a complete excision would have only been possible with a pleuropneumonectomy, which the 83-year-old patient would have not survived. The careful removal of the hematoma along with the mobilization and a partial decortication of the lung, dissection and control of all lobar pulmonary arteries and bronchi, cauterization of the bronchial arteries, and full reex- pansion of the lung with a longer intubation period and an intensive physiotherapy immediately postoperatively is the best solution in these elderly patients.

To our knowledge this is the first report of an elderly patient who was treated successfully for an intrathoracic expanding hematoma by cauterization of the bronchial arteries, an older method used in cases of pulmonary bronchiectasis.

\section{References}

1. Hirai S, Hamanaka Y, Mitsui N, Isaka M, Kobayashi T. Chronic expanding hematoma in the pericardial cavity after cardiac surgery. Ann Thorac Surg. 2003;75:1629-31.

2. Okubo K, Okamoto T, Isobe J, Ueno Y. Rupture of chronic expanding hematoma of the thorax into the lung parenchyma. J Thorac Cardiovasc Surg. 2004;127:1838-40.

3. Hwang GL, Moffatt SD, Mitchell JD, Leung AN. Chronic expanding hematomas of the thorax. AJR Am J Roentgenol. 2003;180:1182-3.

4. Hanagiri T, Murunaka H, Hashimoto M, Nishio T, Sakai S, Ono M, et al. Chronic expanding hematoma in the chest. Ann Thorac Surg. 1997;64:559-61.

5. Labadie EL, Glover D. Physiopathogenesis of subdural hematomas. Part I: Histological and biochemical comparisons of subcutaneous hematomas in rats with subdural hematoma in man. J Neurosurg. 1976 45:382-92.

\title{
Surgical exclusion of a thrombosed azygos vein aneurysm causing pulmonary embolism
}

\author{
Yoshitsugu Nakamura, MD, Kiyoharu Nakano, MD, Hayao Nakatani, MD, Takuya Fukuda, MD, Kentaro Honda, MD,
} and Nobuyuki Homma, MD, Tokyo, Japan

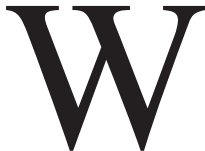

e report a surgical case of a large thrombosed azygos vein aneurysm causing severe pulmonary embolism. The aneurysm diminished spontaneously by closing its connection with the superior vena cava.

\section{Clinical Summary}

A 37-year-old woman was admitted with chest pain and palpitations. A superior vena caval aneurysm had been diagnosed 2 years ago during the patient's left thyroid lobe resection. Her medical

From the Department of Cardiovascular Surgery, Kanto Medical Center NTT EC, Tokyo, Japan.

Received for publication Oct 17, 2006; accepted for publication Nov 9, 2006.

Address for reprints: Yoshitsugu Nakamura, MD, Department of Cardiovascular Surgery, Kanto Medical Center NTT EC, 5-9-22 Higashigotanda, Shinagawa-ku, Tokyo 141-8625, Japan (E-mail: nakamura@kmc.mhc.east. ntt.co.jp).

J Thorac Cardiovasc Surg 2007;133:834-5

$0022-5223 / \$ 32.00$

Copyright $\odot 2007$ by The American Association for Thoracic Surgery

doi:10.1016/j.jtcvs.2006.11.012 history was unremarkable except for thyroid disease. Furthermore, no significant trauma was recorded. Her cardiac, renal, and hepatic functions were normal. A chest radiograph showed mediastinal enlargement occupying the right upper lung field (Figure 1, A). Computed tomography (CT) revealed a huge thrombosed saccular aneurysm of $11 \times 9 \mathrm{~cm}$ at the azygos vein arch (Figure $2, A$ ). The thrombus protruded into the superior vena cava (SVC), and bilateral massive pulmonary embolism was detected (Figure 2, B). Medical therapy for anticoagulation was not effective. Therefore, an emergency operation was performed. Through a median sternotomy, a cardiopulmonary bypass was established by right femoral and left brachiocephalic vein drainage cannulations and an ascending aortic return cannulation. The SVC was snared and intercepted at the junction to the right atrium and left brachiocephalic vein. The SVC was longitudinally incised. The orifice of the azygos vein was $2 \times 2 \mathrm{~cm}$ on the posterior wall of the SVC. The protruding thrombus was removed from the SVC. The orifice was closed with a 4-0 polypropylene running suture. The aneurysm itself was not removed. The right pulmonary artery was incised, and the embolectomy was completed. The postoperative course was uncomplicated. Four years after the operation, the shadow of the aneurysm disappeared on chest radiograph (Figure 1, B), and the aneurysm diminished to $15 \mathrm{~mm}$ in diameter on CT. 

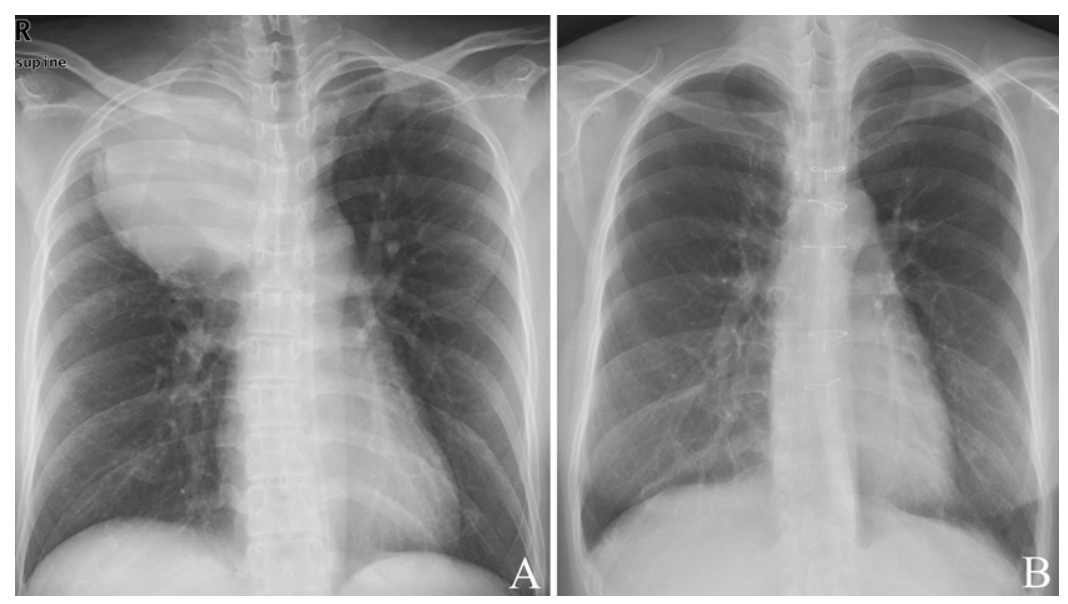

Figure 1. Chest radiography. A, Before operation, an azygos vein aneurysm occupying the right upper lung field. B, Four years after operation, the shadow of the aneurysm has disappeared.
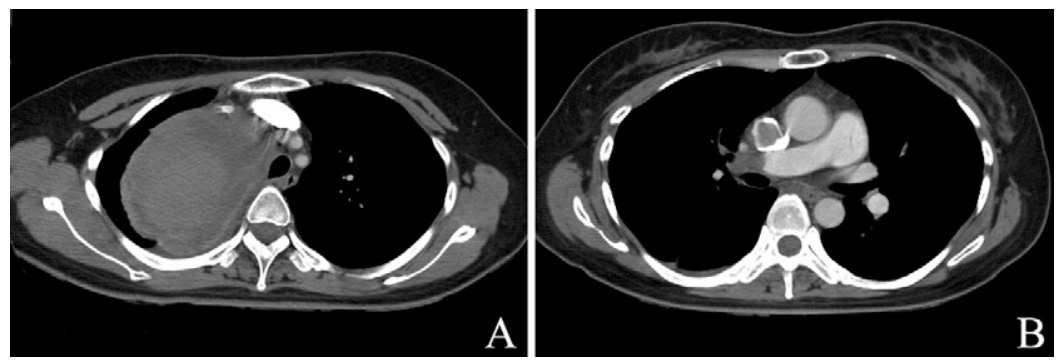

Figure 2. CT. A, Huge saccular aneurysm of $11 \times$ $9 \mathrm{~cm}$ at the azygos vein. B, Thrombus projecting into the SVC and massive pulmonary embolism.

\section{Discussion}

Aneurysm of the azygos vein is rare. ${ }^{1}$ Most reported cases have no specific symptoms. ${ }^{1,2}$ To the best of our knowledge, pulmonary embolism occurring in this exceptional aneurysm has never been reported.

There are 3 main causes of azygos vein aneurysm. First, there is azygos vein dilatation caused by pressure and volume load, such as portal hypertension or inferior vena caval occlusion; this type of aneurysm is usually fusiform. ${ }^{3}$ Second, there is traumatic pseudoaneurysm, including blunt injury or catheter insertion into the azygos vein. Third, there is idiopathic saccular aneurysm; this is assumed to be a congenital aneurysm that is caused by the development of a remnant of an embryologic vein connecting the transverse part of the azygos vein. We believe our case was idiopathic, that is, congenital from the viewpoint of its form and medical history.

The treatment strategy is not clear in azygos venous aneurysm. Most cases of saccular aneurysm have been reported to be removed through a conventional right thoracotomy or thoracoscopic approach. ${ }^{4}$ In our case with pulmonary embolism, a median sternotomy was mandatory for pulmonary embolectomy. The aneurysm was large and had some branches that were difficult to occlude through a median sternotomy. Therefore, we simply excluded the aneurysm from the SVC by closing its orifice to prevent the recurrence of pulmonary embolism. We believe that surgical removal of venous aneurysms is not always necessary because the venous aneurysm is in a low-pressure system. In fact, follow-up with CT for 4 years revealed a significant spontaneous reduction in size of the aneurysm.

One important point is the differential diagnosis of a neoplastic tumor of the azygos vein, for example, leiomyosarcoma. ${ }^{5}$ Definite evaluation by contrast-enhanced CT helps to distinguish between these 2 masses. If vascular neoplasma is suspected by preoperative $\mathrm{CT}$, the mass should be completely removed.

An azygos vein aneurysm is extremely uncommon; however, if it is thrombosed, surgical therapy is necessary to prevent pulmonary embolism. The exclusion technique of the aneurysm can be 1 surgical option.

\section{References}

1. Gallego M, Mirapeix RM, Castaner E, Domingo C, Mata DJ, Marin A. Idiopathic azygos vein aneurysm: a rare cause of mediastinal mass. Thorax. 1999;54:653-5.

2. Gomez MA, Delhommais A, Presicci P, Besson M, Roger R, Alison D. Partial thrombosis of an idiopathic azygos vein aneurysm. Br J Radiol. 2004;77:342-3

3. Lee SY, Kuo HT, Peng MJ, Lin FJ, Shih SC, Sheu CY, et al. Azygos vein varix mimicking mediastinal mass in a patient with liver cirrhosis. Chest. 2005;127:661-4.

4. Person TD, Komanapalli CB, Chaugle H, Schipper PH, Sukumar MS. Thoracoscopic approach to the resection of an azygos vein aneurysm. J Thorac Cardiovasc Surg. 2005;131:230-1.

5. Dasika U, Shariati N, Brown JM. Resection of a leiomyosarcoma of the azygos vein. Ann Thorac Surg. 1998;66:1405. 